\title{
THE PREVALENCE OF LONG-TERM ORAL ANTICOAGULATION THERAPY IN A CARDIOLOGY CENTER IN BUCHAREST, ROMANIA
}

\author{
ADELINA-MIHAELA SORESCU ${ }^{1}$, TUDOR ENACHE, \\ SUZANA GUBERNA ${ }^{1,2}$
}

\author{
${ }^{1}$ Carol Davila University of Medicine and Pharmacy, Bucharest, Romania \\ ${ }^{2}$ Bagdasar-Arseni Emergency Clinical Hospital, Bucharest, Romania
}

\begin{abstract}
Backgroundand aims. Fewstudies discuss the prevalence of oral anticoagulation therapy (OAT) in clinical practice, despite their increasing use worldwide. In America, studies established that $20 \%$ to $80 \%$ of the patients with indication benefit from OAT. In Romania, there is no data regarding the utilization of oral anticoagulants. Thus, this study aims to determine the trends of OAT.

Methods. We designed a cross-sectional study of the patients admitted to the Cardiology Department of the "Bagdasar-Arseni" Clinical Emergency Hospital, Bucharest, from the 1st of November 2016 until the 31st of January 2017. We considered $O A T$ indications to be: atrial fibrillation/flutter (AF), pulmonary embolisms (PE), deep vein thrombosis (DVT), intramural or intracavitary thrombi and left ventricle aneurysms. Statistical analysis was performed with EpiInfo.

Results. There were 783 patients admitted, 253 of these having an OAT indication (mean age 73.25 years, $53.75 \%$ female). Only 162 patients (64.03\%) received it, either Vitamin K Antagonists (VKA) (78 patients, 48.14\%), or Novel Oral Anticoagulants (NOAC) (84 patients, 51.85\%). Reasons for not indicating such therapy included the hemorrhage risk (43.27\%), the lack of adherence to the treatment $(18.56 \%)$, the impossibility of INR monitoring (21.84\%), the economic status $(10.21 \%)$ and others (6.12\%). 221 patients had AF (87.35\%), 141 (63.8\%) receiving OAT, VKA (67 patients, 47.51\%), or NOAC (74 patients, 52.48\%). 17 patients (6.71\%) had a PE and/or DVT. $15(88.23 \%)$ received OAT, AVK (11 patients, $73.33 \%)$, or NOAC (4 patients, 26.67\%). 15 patients (5.92\%) had other OAT indications (excepting AF or PE/DVT), 11 receiving OAT (73.33\%), AVK (8 patients, 72.72\%), or NOAC (3 patients, 27.27\%).

Conclusions. Our study determined that $64.03 \%$ of those with indication received OAT. Similar data is reported in the USA, suggesting an underuse of anticoagulants. The risk of hemorrhage, lack of adherence, the impossibility of INR monitoring or the economic status were some of the reasons for not recommending OAT.
\end{abstract}

Keywords: oral anticoagulation therapy, atrial fibrillation, pulmonary embolism, deep vein thrombosis

\section{Background and aims}

Thromboembolic risks are always present in patients diagnosed with atrial fibrillation or flutter (AF), deep vein thrombosis (DVT), or other cardiovascular afflictions. If we include cerebral vascular accidents (CVAs) or myocardial infarctions on the list of thromboembolic events, we are

Manuscript received: 03.07.2017

Received in revised form: 19.09.2017

Accepted: 24.10.2017

Address for correspondence: adelina_sorescu1904@yahoo.com safe to say that this group of conditions is one of the most prevailing causes of morbidity and mortality worldwide [1].

Compared to a control group, it was found that in patients diagnosed with AF the possibility of developing a CVA is five times higher [2].

For a significant amount of time, vitamin $\mathrm{K}$ antagonists (VKAs), for example warfarin, have been the only prevention and therapeutic option for thromboembolisms. Their effectiveness has been constantly 
proven in literature [3]. However, ever since the novel oral anticoagulants (NOACs) have been approved by the regulatory institutions, their assumed superior effects and the fact that they do not necessitate periodical monitoring have determined their increasing use, in the detriment of VKAs [1]. Depending on their action mechanisms, NOACs can be either thrombin inhibitors (for example dabigatran), or direct factor Xa inhibitors (for example apixaban and rivaroxaban).

In spite of their increasing use among certain patients, relatively few studies actually discuss in literature the prevalence of OAT in clinical practice. In the United States of America (USA), different studies have reported rates ranging from $20 \%$ to $80 \%$ of prescribing OAT to highrisk patients for thromboembolic events, diagnosed with $\mathrm{AF}$ [1]. This therapy is contraindicated in the case of prior bleeding, high-risk for bleeding or previous intracranial hemorrhage, patient refusal, comorbid illnesses, allergies, pregnancy and others $[4,5]$. Thus, the OAT is contraindicated in almost $15 \%$ of the patients with $\mathrm{AF}$ and other cardiovascular conditions [1].

Having in mind the facts presented above, we can say that ever since oral anticoagulants have been approved by the Food and Drug Administration (FDA) and the European Medication Agencies (EMEA), high rates of under-treatment with antithrombotic therapies have been reported in literature.

While no data regarding the utilization of oral anticoagulants has been reported in Romania, Europebased studies have been published. For example, Larsen et al. concluded that in the case of AF, NOACs were used equally $(48.5 \%)$ or more frequently $(33.3 \%)$ than VKAs [6]. Thus, the present study aims to determine the trends of OAT in our country, namely we tried to establish the prevalence of oral anticoagulants among patients with high thromboembolic risks, the main types of OAT most commonly used in clinical practice and the main reasons for not recommending OAT in certain patients. At the end of the study we compared our results to those presented in literature.

\section{Materials and methods}

In order to fulfill our purpose, we designed a descriptive, cross-sectional study. The subjects were selected from the patients admitted to the Cardiology Department at the "Bagdasar-Arseni" Clinical Emergency Hospital, during a three month period of time (from the 1st of November 2016 until the 31st of January 2017). The inclusion criteria represented OAT indications, meaning that we included in our study patients diagnosed with atrial fibrillation or flutter (AF), pulmonary embolisms (PE), deep vein thrombosis (DVT), intramural or intracavitary thrombi and left ventricle aneurysms (Figure 1).

Information regarding each patient was collected from their personal medical records. We noted their age,

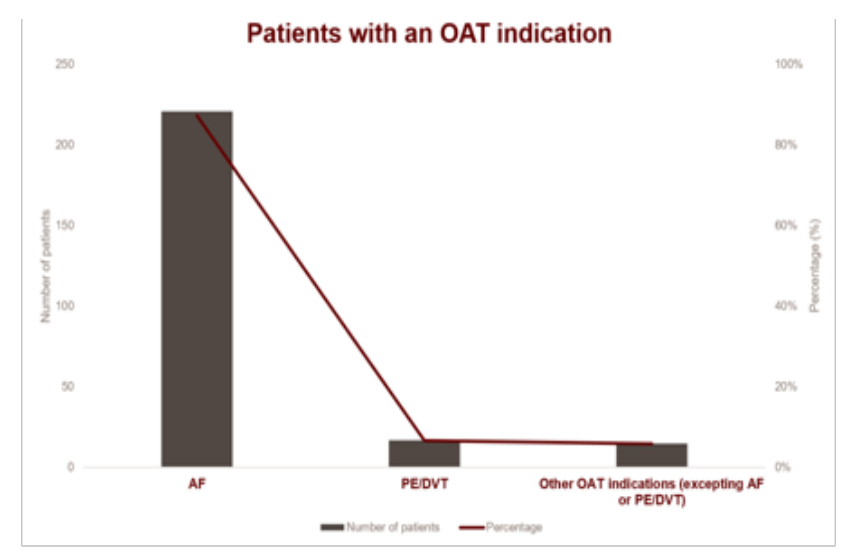

Figure 1. The distribution of OAT indications (grey columns represent the number of patients, while the red line represents the percentage of patients). It can be observed that $\mathrm{AF}$ is the most prevalent condition which requires long-term oral anticoagulation.

gender, diagnoses, whether they received or not OAT and what oral anticoagulant was used in each case. If no long-term oral anticoagulant was prescribed, we noted the reasons for not recommending this therapy.

We divided the patients into groups, according to their diagnosis. For each group, we determined the frequency of OAT in those with an indication for such therapy. Moreover, we determined the most commonly used type of oral anticoagulant, either VKAs or NOACs. Apart from these, we determined the recurring reasons employed for not prescribing long-term OAT. Statistical analysis was performed with EpiInfo.

We compared our results with those reported in literature. Therefore we performed a PubMed search for similar studies. Four studies discussing the prevalence of OAT in the United States of America were found. We found no studies which discussed the use of oral anticoagulants in Europe, or Romania.

\section{Results}

During the analyzed period of time, 783 patients were hospitalized in the Cardiology Department. We collected pieces of information only from the patients having an OAT indication. Thus, our study included 253 patients, representing $32.31 \%$ (mean age 73.25 years, $53.75 \%$ female). We tried to determine each patient's anticoagulation status. From the group with OAT indication, 167 patients (66.01\%) received the therapy. These patients received either vitamin $\mathrm{K}$ antagonists (VKA) (80 patients, $47.90 \%$ ), or novel oral anticoagulants (NOAC) (87 patients, $52.09 \%$ ).

The majority of the patients, 221 ( $87.35 \%$ of the OAT indications), were included in the AF group. Only 63.8\% (141 patients) of these actually received oral anticoagulants (Figure 2). Either VKAs or NOACs were used. While 67 patients $(47.51 \%)$ received VKAs, the rest of the patients (74 patients, $52.48 \%$ ) received NOACs (Figure 3 ). 


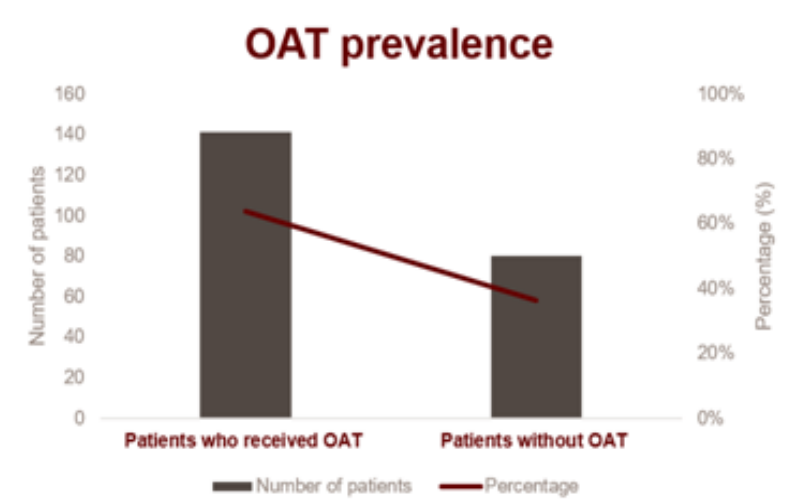

Figure 2. The number and percentage, respectively, of patients who received or not OAT.

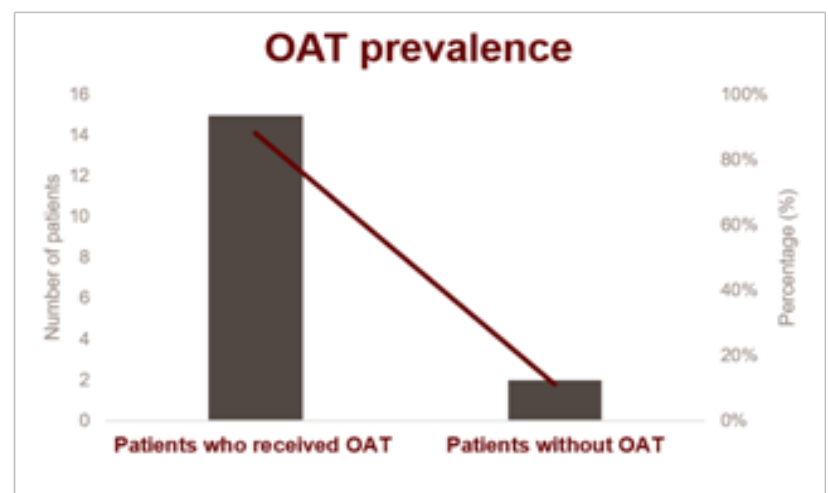

Figure 4. The prevalence of OAT in patients diagnosed with DVT and/or PE.

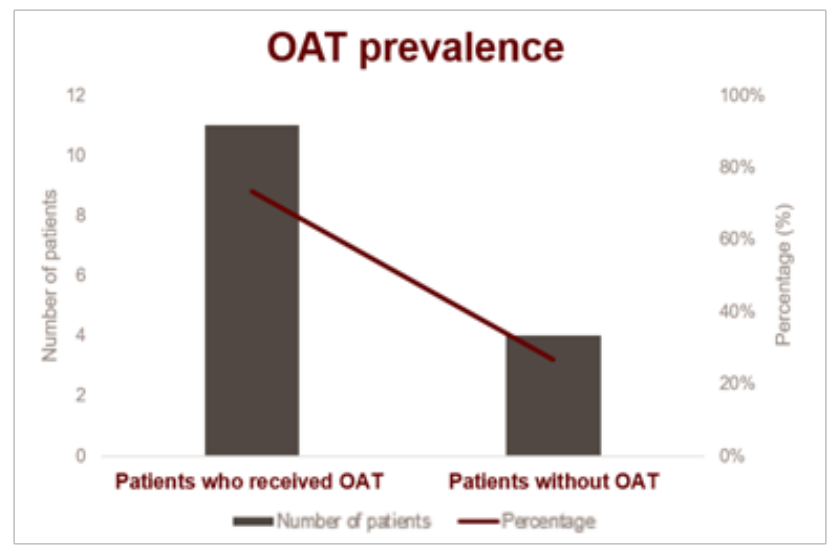

Figure 6. The prevalence of OAT among patients with other indications for oral anticoagulation, other than AF or DVT/PE.

The group with PE and/or DVT was less represented, including only 17 patients ( $6.71 \%$ of the OAT indications); 15 of these patients, meaning $88.23 \%$, received longterm anticoagulation therapy (Figure 4). Most of them, 11 patients $(73.33 \%)$ received VKAs, while the rest of them (4 patients, 26.67\%) had been prescribed NOACs (Figure 5).

Other OAT indications, excepting AF or PE/DVT (including intramural or intraventricular thrombi and left ventricle aneurysms) added up to 15 patients $(5.92 \%$ of

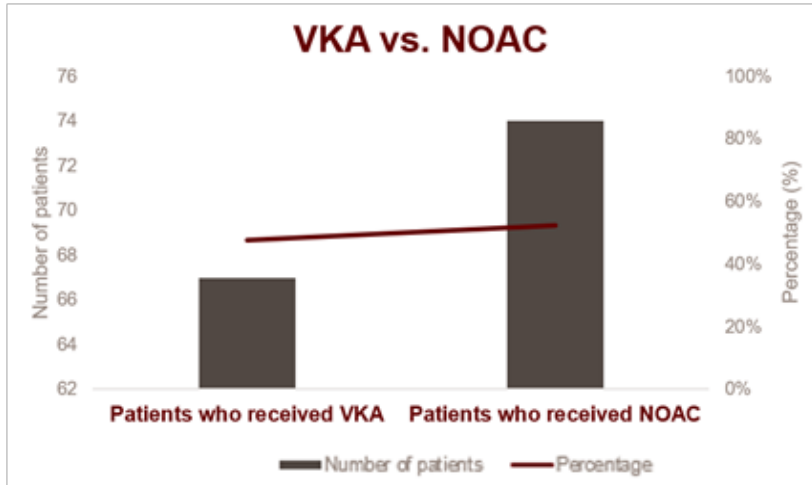

Figure 3. The types of oral anticoagulants prescribed to patients diagnosed with AF.

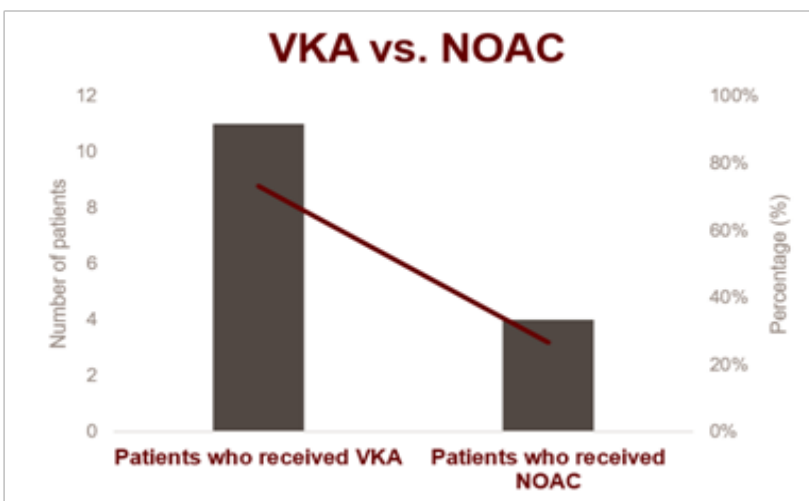

Figure 5. The types of oral anticoagulation used in patients with DVT/PE.

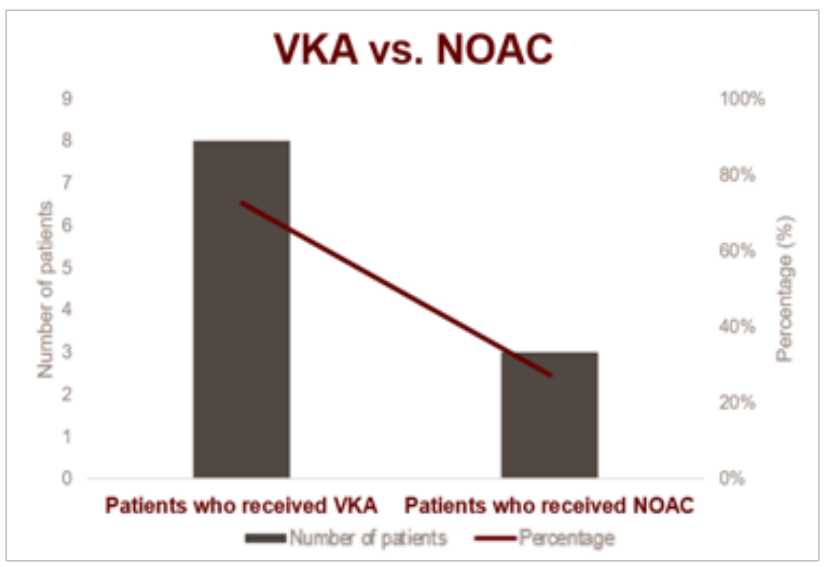

Figure 7. The types of oral anticoagulants used in patients with other indications for oral anticoagulation, other than AF or DVT/PE.

the OAT indications). From these, 11 patients received oral anticoagulants $(73.33 \%$ ) (Figure 6). VKAs were most frequently used, 8 patients $(72.72 \%)$ receiving it. Meanwhile, NOACs were prescribed only to 3 patients (27.27\%) (Figure 7).

Apart from the OAT contraindications, there are cases when such therapies are not recommended. Only 86 of the patients who had an OAT indication did not receive it. The most common reasons for not indicating such 
therapy included the hemorrhage risk (37 patients, meaning $43.27 \%$ ), the impossibility of INR monitoring combined with the poor economic status (28 patients, $32.05 \%$ ), the lack of adherence to the treatment (16 patients, $18.56 \%$ ), and others (5 patients, 6.12\%) (Figure 8 ).

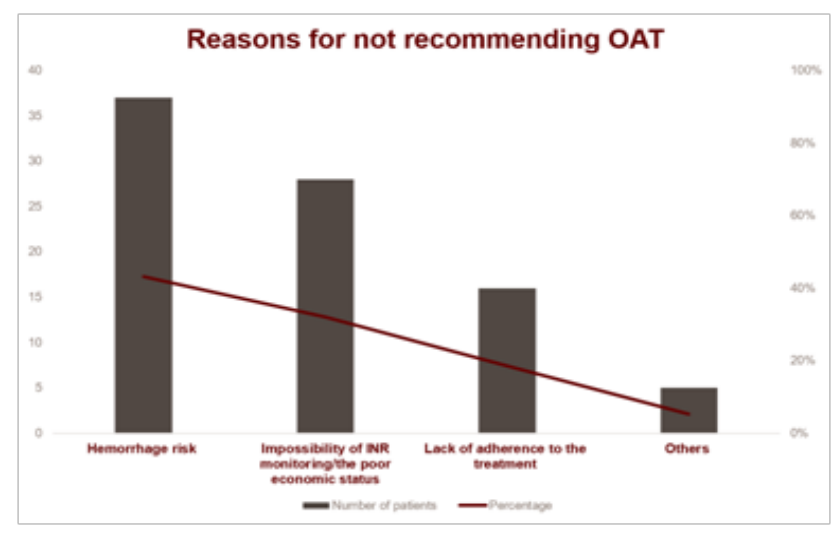

Figure 8. The reasons employed for not recommending OAT, even though an indication for this therapy existed.

\section{Discussion}

Thromboembolic events are an increasingly frequent problem worldwide. That is the reason for trying to prevent and treat such conditions. AF, DVT, intraventricular thrombi are some of the cardiovascular conditions that increase the risk of developing thromboembolism.

The role of oral anticoagulation therapy (OAT) in reducing the prospects of developing thromboembolisms has been discussed in literature. For example, the use of VKAs decreases the possibility of developing a CVA by almost $30 \%$ in the patients at risk for thromboembolic events [7].

In our study we tried to determine the prevalence of oral anticoagulation in a Cardiology Department in Romania. By comparing our results with the national trends in the United States of America, we found an underuse of oral anticoagulants, similar to the USA [1]. We found that $66.01 \%$ of the patients with an indication for OAT actually receive this medication, a percentage that is included in the rates reported in literature, namely $20-80 \%$ [1]. What is more, the IMS Health National Disease and Therapeutic Index suggested that ever since the introduction of NOACs in 2010, the use of these drugs has increased, making them the dominant option in OAT [1]. Similar to these facts, we found that while VKAs were prescribed to $47.90 \%$ of the patients in the study, NOACs were present in $52.09 \%$ of the cases.

In the case of $\mathrm{AF}$, we found that $63.8 \%$ of the diagnosed patients were in fact prescribed OAT. Our findings were below the average rates reported in literature. Studies suggest that in developed countries $65 \%$ to $80 \%$ of the patients diagnosed with $\mathrm{AF}$ and a CHADS2 or CHA2DS2VASc risk scores higher than 2, receive long- term OAT $[8,9]$. Studies have tried over the years to determine the best OAT option for AF. NOACs have a number of advantages over warfarin. Not needing to monitor the NOAC administration, the reduced presence of interaction with other drugs and food and most importantly the reduced incidence of hemorrhage are only some of the reasons mentioned in literature for choosing NOACs over VKAs [10]. The increasing rates of utilization of NOACs, used in the AF therapeutic scheme, have been seen in numerous studies [1]. Our findings were consistent with the literature. That is to say, VKAs were used in $47.51 \%$ of the cases, while the rest received NOACs $(52.48 \%)$.

Speaking about DVT and/or PE, a higher use of oral anticoagulants than in the case of AF was seen. $88.23 \%$ of the patients diagnosed with DVT and/or PE received OAT. This high rate of oral anticoagulation use may be determined by the higher risk of thromboembolic events following a DVT. Contrary to other studies, VKAs $(73.33 \%)$ were more frequently used than NOACs $(26.67 \%)$. It has been concluded in literature that longterm NOAC administration is a better treatment option for patients with DVT or PE [11]. However, possible limitations in the anticoagulant use have been suggested. For example, Yeh et al., concluded in 2014 that the lack of antidotes for NOACs and the impossibility of using them in patients with kidney disease are some aspects to take into consideration when prescribing these drugs [10,12].

A majority - 73.33\% of the patients with other OAT indications (excepting AF or PE/DVT) received longterm OAT. Similar to the case of PE/DVT, VKAs were the dominant treatment option $(72.72 \%$ of the patients received VKAs, in contrast to the NOACs, which were used in $27.27 \%$ of the cases).

It has been concluded in literature that OAT is contraindicated in almost $15 \%$ of the patients with AF and other cardiovascular conditions [1]. In spite of that, in our center, 33.99\% did not receive long-term OAT. Several reasons were given for not recommending such treatment. The most prevalent one was the risk of hemorrhage, present in $43.27 \%$ of the cases. However, studies showed a reduced hemorrhage risk when using apixaban, comapared to other NOACs or warfarin [10]. Other frequent motives for not prescribing oral anticoagulants to patients with such indication were the impossibility of INR monitoring combined with the poor economic status $(32.05 \%$ of the cases). These might be the reasons for the lower use of NOACs in the case of DVT/PE, intracavitary thrombi and ventricular aneurysms. The lack of adherence to the treatment (18.56\% of the cases), and other reasons were also found in the patients' medical records.

\section{Conclusions}

The main OAT indications include atrial fibrillation/ flutter, pulmonary embolisms, deep vein thrombosis, intramural or intracavitary thrombi and left ventricle 


\section{aneurysms.}

Our study determined that $66.01 \%$ of those with indication received OAT. Similar data are reported in the USA, most of the studies in literature suggesting an underuse of anticoagulants.

The risk of hemorrhage, lack of adherence, the impossibility of INR monitoring or the poor economic status were some of the reasons for not recommending OAT, the risk of hemorrhage being the dominant reason.

\section{References}

1. Kirley K, Qato DM, Kornfield R, Stafford RS, Alexander GC. National trends in oral anticoagulant use in the United States, 2007 to 2011. Circ Cardiovasc Qual Outcomes. 2012;5(5):615-621. 2. Wolf PA, Abbott RD, Kannel WB. Atrial fibrillation as an independent risk factor for stroke: the Framingham Study. Stroke. 1991;22(8):983-988.

3. Ageno W, Gallus AS, Wittkowsky A, Crowther M, Hylek EM, Palareti G. Oral anticoagulant therapy: Antithrombotic Therapy and Prevention of Thrombosis, 9th ed: American College of Chest Physicians Evidence-Based Clinical Practice Guidelines. Chest. 2012;141(2 Suppl):e44S-e88S.

4. O'Brien EC, Holmes DN, Ansell JE, Allen LA, Hylek E, Kowey $\mathrm{PR}$, et al. Physician practices regarding contraindications to oral anticoagulation in atrial fibrillation: findings from the Outcomes Registry for Better Informed Treatment of Atrial Fibrillation (ORBIT-AF) registry. Am Heart J. 2014;167(4):601-609.e1.

5. Steinberg BA, Greiner MA, Hammill BG, Curtis LH, Benjamin EJ, Heckbert SR, et al. Contraindications to anticoagulation therapy and eligibility for novel anticoagulants in older patients with atrial fibrillation. Cardiovasc Ther. 2015;33(4):177-183.

6. Larsen TB, Potpara T, Dagres N, Proclemer A, Sciarrafia E, Blomström-Lundqvist $\mathrm{C}$, et al. Preference for oral anticoagulation therapy for patients with atrial fibrillation in Europe in different clinical situations: results of the European Heart Rhythm Association Survey. Europace. 2015;17(5):819-824.

7. Bartholomay E, Polli I, Borges AP, Kalil C, Arroque A, Kohler I, et al. Prevalence of oral anticoagulation in atrial fibrillation. Clinics (Sao Paulo). 2014;69(9):615-620.

8. Chae SH, Froehlich J, Morady F, Oral H. Prevalence and predictors of warfarin use in patients with atrial fibrillation at low or intermediate risk and relation to thromboembolic events. Clin Cardiol. 2011;34(10):640-644.

9. Nilsson GH, Björholt I, Krakau I. Anticoagulant treatment of patients with chronic atrial fibrillation in primary health care in Sweden--a retrospective study of incidence and quality in a registered population. Fam Pract. 2004;21(6):612-616.

10. Shafeeq $H$, Tran TH. New oral anticoagulants for atrial fibrillation: are they worth the risk? P T. 2014;39(1):54-64.

11. Cohen AT, Hamilton M, Bird A, Mitchell SA, Li S, Horblyuk R, et al. Comparison of the Non-VKA Oral Anticoagulants Apixaban, Dabigatran, and Rivaroxaban in the Extended Treatment and Prevention of Venous Thromboembolism: Systematic Review and Network Meta-Analysis. PLoS One. 2016 Aug 3;11(8):e0160064. doi: 10.1371/journal.pone.0160064.

12. Yeh CH, Gross PL, Weitz JI. Evolving use of new oral anticoagulants for treatment of venous thromboembolism. Blood. 2014;124(7):1020-1028. 